\title{
Dexmedetomidine as an anesthetic adjuvant to control intraocular pressure: a meta-analysis
}

\author{
Chengmao Zhou ${ }^{1}$, Yuting Yang ${ }^{2}$, Zhen Liu ${ }^{1}$, Lin Ruan ${ }^{1 *}$ \\ ${ }^{1}$ Department of Anesthesiology, the Affiliated Tumor Hospital of Guangxi Medical University, \\ Nanning 530021, China . \\ ${ }^{2}$ Department of Chemotherapy, the Affiliated Tumor Hospital of Guangxi Medical University, \\ Nanning 530021, China . \\ RuanLin187@163.com.
}

Keywords: Dexmedetomidine; Intraocular pressure; Hemodynamic effects; Meta-analysis

\begin{abstract}
The aim of this meta-analysis was to evaluate the effect of dexmedetomidine on intraocular pressure (IOP) and hemodynamict.

Methods: There were 12 randomized clinical trials in the meta-analysis. The Primary outcomes were the Weighted Mean Difference (WMD) and the 95\% Confidence Intervals (CI) of changing IOP compared dexmedetomidine with placebo. And the second outcomes were the Standardized Mean difference (SMD) 、 the Risk Ratio (RR) and the 95\% Confidence Intervals (CI).

Results: Twelve trials totaling 702 patients were analyzed. In the two key point time of the anesthesia administration, the pooled estimates favored lowering effect of dexmedetomidine on IOP when compared with placebo (WMD $=-4.47 \mathrm{mmHg}$, 95\% CI (-6.97,-1.96), P<0.00001; and WMD $=-2.13 \mathrm{mmHg}, 95 \% \mathrm{CI}(-3.51,-0.75), \mathrm{P}=0.003$, respectively) . Dexmedetomidine also hastened the block onset time (WMD $=-0.61$ minute, 95\% CI $(-0.90,-0.33), \mathrm{P}<0.0001)$. And there was no significant difference of hypotension between dexmedetomidine and placebo $(\mathrm{RR}=1.12,95 \% \mathrm{CI}$ $(0.47,2.64), \mathrm{P}=0.80)$. However, for the point of the incidence of bradycardia, the use of dexmedetomidine could increase it when compared with placebo $(\mathrm{RR}=7.50,95 \% \mathrm{CI}(1.76,31.80)$, $\mathrm{P}=0.006)$.
\end{abstract}

\section{Introduction}

Glaucoma is the second-ranking cause of blindness in the global world, there will be increased from 60.5 million people in 2010 to 79.6 million in 20201. A meta-analysis has shown that lowering intraocular pressure (IOP) could reduce the risk of glaucoma 2. And there is a close relationship between the change of IOP and many orbital barriers, including genetic, structural, inflammation, trauma, tumor diseases 3.Especially in ophthalmic surgery, the factor, which could increase the IOP, may lead a permanent damage of vision4. And the anesthesia manipulates such as tracheal extubation 5had a strong stimulus that may elevate the IOP[1]. So the anesthesia administration of ophthalmic surgery should provide a lower IOP and stable hemodynamics6.

Dexmedetomidine is a novel drug, and it could improve anesthesia management in various operations7 because of its unique characteristics. An early basic study has shown that IOP in rabbits was reduced by dexmedetomidine8. After that, a lot of related human study began to carry out. And from much of the literature of several clinical trials, it seems that Dexmedetomidine could reduce the IOP, but the conclusions were conflicted.

The aim of this meta-analysis is to evaluate the effect of dexmedetomidine on IOP and hemodynamic effects. There was no quantitative or qualitative review on this focus has been published before, although we tried our best to search it.

\section{Materials and methods}

Search Strategy and Trials Selection. The clinical trials included in this meta-analysis were searched from the following databases: PubMed, Ovid, EMBASE, Cochrane Central Register of 
Controlled Trials, and Web of Science(January1985-October 2014). Other sources were from China National Knowledge Infrastructure Database (CNKI) and Google scholar. Mesh terms and key words used for the searches included "dexmedetomidine" and "intraocular pressure". Meanwhile, the references of included studies were reviewed for additional trials. The last search was conducted in October 2015. No language restrictions were placed on the search strategies and trial inclusion.

Inclusion and exclusion criteria. Published clinical trials were eligible for this meta-analysis, which had to meet the protocol-determined inclusion criteria: (1) studies in comparison of the use of dexmedetomidine and placebo or other drugs on the intraocular pressure were randomized controlled trials (RCTs); (2) the research objects should be human beings of any sex and age; (3) there were no restrictions on dosing regimen and the administration of dexmedetomidine; (4) the available outcome data was provided.

The extraction criteria were as follows: (1) abstracts, duplicate publications and reviews; (2) did not report the specific results ; (3) not full text.

Data extraction and quality assessment. Two authors extracted the data from the included studies independently (Chengmao Zhou, Yuting Yang). The following data were extracted from each trial: author, year of publication, age, the type of operation, the baseline of IOP, intervention measures and so on[2]. For a study, including subjects of three groups (dexmedetomidine, placebo and other drugs), data were extracted from dexmedetomidine and placebo. When the dexmedetomidine doses in a trial were differed, only the data of highest dexmedetomidine and placebo group were extracted 9.

The primary outcome was mean IOP(two time point :one minute after intubation and after pretreatmen)with standard deviation (SD). The secondary outcomes included mean arterial pressure (MAP) and heart rate (HR) in the time point of one minute after induction, hypotension, and bradycardia.

Two authors (Chengmao zhou, Yuting Yang) and a third author (Lin Ruan) independently assessed risk of bias for each research according to the 4 items Oxford scale10,which including the reporting and adequacy of randomization( 0 to 2 points), allocation concealment( 0 to 1 points $)$, double blinding( 0 to 2 points), and description of drop-outs( 0 to 2 points)[3]. The items are summed, with summary scores varying from 0 to 7 .

Statistical analysis. The Cochrane Collaboration's statistical software (RevMan 5.0) was used for data input, statistical analysis, and creation of graphs.

Classification results are assessed with a risk ratio (RR) and 95\% confidence interval (CI), and continuous results are weighted with mean difference (WMD) or Standardized Mean difference (MD). It is considered $\mathrm{p}<0.05$ as a statistically significant. Heterogeneity was assessed by the I2 test. If the outcome data was low heterogeneity (I2 $<50 \%$ ), the fixed-effect model was chosen. If the outcome data was high heterogeneity (I2 > 50\%), the random-effect model was used to analysis it.

The evidence from the results of fixed-effect model and random-effects model should not be changing significantly.

\section{Results}

Included studies. A total of 150 studies were relevant to the search terms after searching. 17 duplicate records and 116 irrelevant records were removed. Another 5 articles were excluded for the following reason: (1) five studies11-16 did not report the specific results comparing dexmedetomidine with placebo (their results all favored lowering effect of dexmedetomidine on IOP when compared with placebo)[4]. Finally, only 12 articles17-28 were identified. The flow chart for the procedure is shown in Figure 1.(Figure 1)

Study and participant characteristics and methodological quality. The basic characteristics and methodological quality of the included RCT are described in Table1.

This meta-analysis reviewed the trials included a total of 702 patients: 351 patients in the dexmedetomidine group and 351 in the control group. The participation of local anesthetics was existed in two trials27, 28. The dose of dexmedetomidine was not all in the same. 
As is known to all, the two time points of "After induction 1 minute" and "After pretreatment of dexmedetomidine" are the key time of anesthesia administration, so we only discuss IOP in these time points.

Heterogeneity inspection and sensitivity analysis. After using the Review Manager (version 5.1, 2011), heterogeneities were in the meta-analysis study (Figure 2). So subgroup analysis was made separately. And the groups of suxamethonium 、 midazolam and none of them were made (Figure 2A, the heterogeneity among the pooled studies in this group was significant, I2=0.91). And the groups of adult and children were made (Figure 2B, the heterogeneity among the pooled studies in this group was significant, $\mathrm{I} 2=0.73$ ).

\section{Primary outcomes}

Secondary outcomes. Dexmedetomidine versus placebo for mean arterial pressure (MAP) and heart rate (HR)in the time point of one minute after induction

Two trials18, 25 including 78 patients compared induction MAP in patients treated with dexmedetomidine or placebo in Figure 3.

Meta-analysis showed that a significantly lower induction MAP was associated with dexmedetomidine $(\mathrm{SMD}=17.30 \mathrm{mmHg}, 95 \% \mathrm{CI}(-31.31,-3.30), \mathrm{P}=0.02)$. (Fig. $3 \mathrm{C})$. The heterogeneity among the pooled studies in this group was significant $(\mathrm{I} 2=0.91 ; \mathrm{P}<0.001)$. The random-effect model was selected.

Three trials including 78 patients compared induction HR in patients treated with dexmedetomidine or placebo in Figure $3 \mathrm{D}$.

Meta-analysis showed that a significantly lower induction HR was associated with dexmedetomidine (SMD $=-20.54$ beats/minute, 95\% CI $(-24.65,-16.44), \mathrm{P}<0.00001)$, without statistical evidence of heterogeneity among the studies (I2=0.44; $\mathrm{P}=0.18$ ).

Dexmedetomidine versus placebo for block onset time. Data regarding block onset time were available from two trials reviewed and are presented in Figure 4.

The block onset time was significantly shorter in group dexmedetomidine than in group placebo (SMD $=-0.61$ minute, 95\% CI $(-0.90,-0.33), \quad \mathrm{P}<0.0001)$, without statistical evidence of heterogeneity among the studies ( $\mathrm{I} 2=0 ; \mathrm{P}=0.92)$.

There were no significant heterogeneities, so the fixed-effect model was selected by us.

Dexmedetomidine versus placebo for the cardiovascular events control. Nine trials18-26 including 404 patients compared pharmacological intervention to control the cardiovascular events in patients treated with dexmedetomidine or placebo in Figure 5.

This meta-analysis showed that there was no significant difference in the incidence of the use of dexmedetomidine for hypotension when compared with placebo $(\mathrm{RR}=1.12,95 \% \mathrm{CI}(0.47,2.64)$, $\mathrm{P}=0.80$ ) (Fig. $5 \mathrm{E}$ ), without statistical evidence of heterogeneity among the studies (I2=0; $\mathrm{P}=0.72)$. Whereas there was a significantly higher incidence of treatment for bradycardia $(\mathrm{RR}=7.50$, , 95\% CI $(1.76,31.80), \quad \mathrm{P}=0.006)$ (Fig. $5 \mathrm{~F}$ ) without statistical evidence of heterogeneity among the studies (I2=0; $\mathrm{P}=0.97)$.

There were no significant heterogeneities, so the fixed-effect model was selected by us.

\section{Discussion}

Our meta-analysis of the literature suggested that the use of dexmedetomidine as an adjuvant pharmacological intervention significantly reduced the IOP compared with placebo. For the time point of one minute after induction, IOP, MAP and HR reached statistical significance after use of dexmedetomidine in comparison with that of placebo. Dexmedetomidine also hastened the onset time of block compared with placebo, whereas there were no differences in hypotension between dexmedetomidine and placebo. Moreover, the meta-analysis suggested an increased risk of bradycardia after use of dexmedetomidine in comparison with that of placebo. The advantages of dexmedetomidine reducing IOP might be caused by a likelihood of reducing MAP and HR. 
Dexmedetomidine has passed the United States food and drug administration (FDA) as a short-term sedative (less than 24 hours) and analgesia in emergency care settings29, which was a highly selective alpha 2 adrenergic receptor agonist medicine, previously used for the short-term sedation of critically ill patients with mechanical ventilation 30 . The following explanation may be used to account for the effect of reducing intraocular pressure of dexmedetomidine:1) aqueous humor is conducive to exclude by weakening the vascular tension of sympathetic eye drainage system 31;2) it is also likely to promote the aqueous humor drainage to decrease sympathy mediated vasomotor tone of drainage system8;3 )the reason of reduction of IOP may be the hemodynamic effect of dexmedetomidine. Our meta-analysis also suggested that when dexmedetomidine reduced IOP, the hemodynamic effect was unstable.

However, our sensitivity analysis suggested that when it came to patients with children, the beneficial effect of dexmedetomidine in reducing IOP did not exist. The outcome was similar with the the administration of no midazolam or suxamethonium.

In contrast with placebo, dexmedetomidine significantly reduced the block onset time. A recently published study32 also presented a similar result for the effect of dexmedetomidine on block onset time, in which dexmedetomidine was compared with a placebo on the duration of infraclavicular brachial plexus block. The favorable role of dexmedetomidine in treatment of block onset time is more likely because of $\alpha-2$ agonist. The evidence33 suggests that $\alpha-2$ agonist has a synergic effect with intrathecal bupivacaine, which may decrease the block onset time. Besides, our analysis showed that compared with placebo, dexmedetomidine decreased the block onset time. However, this conclusion about the block onset time by dexmedetomidine was in disagreement with other study34, which showed it could not hasten the onset time.

Although bradycardia and hypotension are common side effects of using the dexmedetomidine in the administration of anestheisa, we could not demonstrate any association between dexmedetomidine and the incidences of hypotension compared with placebo. And a current meta-analysis reported a result: patients treated with dexmedetomidine provide the improvement of hemodynamic stability35. While our meta-analysis suggested that dexmedetomidine was associated with a significant increase of the incidence of bradycardia.

Some clinical limitations in our meta-analysis warrant discussion. First of all, the data of this meta-analysis was just based on the existing literature, although we have tried to search other sources; the conclusion was also based on a relative small number of individual RCTs. Second, another limitation is that the anesthesia drugs such as fentanyl, propofol, sevoflurane, midazolam and suxamethonium also have an effect of reducing or increasing IOP, which may affect the effect of dexmedetomidine on IOP.

In summary, dexmedetomidine may be a promising agent to control IOP because it could offer a shorter block time, stable hemodynamic effects and a good efficacy of reducing IOP. However, there are present data to support the risk of bradycardia using of dexmedetomidine in the clinical setting. In addition, more evidences are to be collected to draw a definite conclusion on the effects of dexmedetomidine on IOP and hemodynamic effects.

\section{Conclusions}

This meta-analysis shows that dexmedetomidine has a lowering effect of IOP and a stable hemodynamic effect. Besides, block onset time will be shorter after the use of dexmedetomidine. However, it can increase the risk of bradycardia.

\section{References}

[1] Byram, M. 1988. Foreign language education and cultural studies. Language, Culture and Curriculum, 1(1), pp.15-31.

[2] Byram, M. 1997. Teaching and Assessing Intercultural Communicative Competence. Clevedon: Muhilingual Matters. 
[3] L. Xie. On Bilingualism, Translation and Professional Translation. Everyone, 2010,17: 230.

[4] W.F. Sun. Acquisition of A Second Language Based on the Language Typology. Language Teaching And Research, 2012,02: 1-8. 